\title{
A Product Match Adjusted R Squared Method for Defining Products with Transaction Data
}

\author{
Antonio G. Chessa ${ }^{1}$
}

\begin{abstract}
The occurrence of relaunches of consumer goods at the barcode (GTIN) level is a well-known phenomenon in transaction data of consumer purchases. GTINs of disappearing and reintroduced items have to be linked in order to capture possible price changes.

This article presents a method that groups GTINs into strata ('products') by balancing two measures: an explained variance (R squared) measure for the 'homogeneity' of GTINs within products, while the second expresses the degree to which products can be 'matched' over time with respect to a comparison period. The resulting product 'match adjusted $\mathrm{R}$ squared' (MARS) combines explained variance in product prices with product match over time, so that different stratification schemes can be ranked according to the combined measure.

MARS has been applied to a broad range of product types. Individual GTINs are suitable as products for food and beverages, but not for product types with higher rates of churn, such as clothing, pharmacy products and electronics. In these cases, products are defined as combinations of characteristics, so that GTINs with the same characteristics are grouped into the same product. Future research focuses on further developments of MARS, such as attribute selection when data sets contain large numbers of variables.
\end{abstract}

Key words: Consumer price index; product relaunch; stratification.

\section{Introduction}

The increased availability of electronic transaction data sets for the consumer price index (CPI) offers possibilities to national statistical institutes (NSIs) to enhance the quality of index numbers. More refined methods can be applied that deal with the dynamics of consumption patterns in a more appropriate way than traditional fixed-basket methods. For instance, multilateral methods can be used to specify monthly weights based on actual sales at the most detailed item level and new items can be directly included in index calculations (De Haan and Van der Grient 2011; Ivancic et al. 2011; Krsinich 2014; Chessa 2016; Chessa et al. 2017; ABS 2017; Diewert and Fox 2017; Van Loon and Roels 2018).

Electronic transaction or scanner data sets contain expenditures and quantities sold of items purchased by consumers at physical or online sales points of a retail chain. The sales data are often aggregated by retailers into weekly sales for each individual item, which is uniquely identified by its Global Trade Item Number (GTIN); that is, the barcode.

\footnotetext{
${ }^{1}$ CPI department, Statistics Netherlands, P.O. Box 24500, 2490 HA The Hague, the Netherlands. Email: ag.chessa@cbs.nl.

Acknowledgments: This research was funded by a grant assigned by Eurostat, for which the author would like to express his gratitude. The author wishes to thank the associate editor and two referees for their comments and useful suggestions, and also colleagues of various NSIs for discussions on this topic.
} 
Transaction data sets also contain characteristics, such as brand and package volume, of the items sold. While traditional price collection methods typically record prices of several tens of items in shops, electronic transaction data sets may contain several tens of thousands of items for a single retail chain.

GTINs represent the most detailed item level in electronic transaction data sets. Each item has a unique barcode. This essentially means that NSIs are given a set of tightly defined items. The ratio of monthly expenditure and quantity sold yields a transaction price, which can be followed for each item from month to month. However, items may be removed from the market and reintroduced with modified packaging, for instance, in order to fit a retailer's new product line. Quality characteristics of such 'relaunch' items may remain the same, but the barcodes may change after reintroduction, and also the prices compared with those under the previous GTINs. The barcodes of the old and new, reintroduced items have to be linked in order to capture price changes under such relaunches.

Typical market segments that feature relaunches are pharmacy items, clothing and electronics. Rates of item churn may reach high levels, since new item assortments are frequently introduced to replace their predecessors. GTINs of relaunch items have to be linked, which means that broader item definitions are needed in such situations. A generic concept is therefore introduced in this article to denote groups of linked items, which is called product. However, a product may also be equivalent to a single item, which is a logical choice in situations where relaunches do not occur.

Old and new GTINs can be linked manually for small samples of items. However, this becomes infeasible when NSIs aim at processing all GTINs each month, or at least those GTINs that account for a high percentage of total expenditure. To date, a method for linking GTINs of relaunch items or, in more general terms, for defining products, that is both broadly applicable and efficient does not appear to exist. Recent studies from different NSIs have shown a need for such a method (Bilius et al. 2018; Hov and Johannesen 2018; Keating and Murtagh 2018). Finding a generic and efficient method is the objective of this article.

Section 2 shows several examples of product types with different dynamics of GTINs that enter and leave an assortment. This section gives an initial, rough impression of the possible impact of different choices with regard to product definition on a price index. The central element of this article, the method MARS for defining products, is described in Section 3. MARS (product Match Adjusted R Squared) has been applied to different types of products, with different rates of churn: food items, clothing, pharmacy products and electronics. Some results are shown in Section 4.

Section 5 treats specific methodological and practical topics concerning product definition. Transaction data sets usually contain a small number of product attributes (often up to four or five in our experience). Are the available attributes sufficient for defining products? An important question from a practical perspective is how MARS could be applied in a production environment. Conclusions are presented in Section 6.

\section{Assortment Dynamics}

As was mentioned in the previous section, certain types of products are affected more by item relaunches than other product types. This section gives several examples with 
different rates of churn. Combining GTINs based on common characteristics is one possible way of linking GTINs of relaunch items. This section also gives an initial impression of the impact of linking versus not linking on a price index, which serves to highlight the importance of the problem of product definition.

The focus in this article is primarily on transaction data. Four product types from data sets of four different Dutch retail chains are considered: milk, cheese and eggs of a supermarket chain, infant garments of a department store chain, hair care products of a pharmacy chain and televisions of an electronics retailer. About four years of data are used for the first two product types and three years of data for the other two product types.

The dynamics of products leaving and entering an assortment over time can be measured in different ways. Chessa et al. (2017) quantify the percentages of existing, leaving and entering products in each month with respect to the preceding month for different types of products. A similar measure is used in this section, which is modified on two points:

(1) The comparison or base month is fixed, and is taken to be the first month of a 13month time window (December of the previous year), and

(2) The share of 'existing products' in the total number of products sold in a month is taken as a measure of assortment dynamics. Existing products are products that are sold both in the base month and in the current month.

These two choices can be translated into the following formal notation. Quantities of an item $i$ sold in month $t$ are denoted by $q_{i, t}$ and $G_{t}$ is the set of items sold in month $t$. The comparison or base month is denoted as month 0 . Let $G_{0, t}$ be the set of items/GTINs that are sold both in the base month and in (current) month $t$. The measure of dynamics proposed in this article does not merely count numbers of products, but quantifies the numbers sold. This choice expresses the extent of churn more appropriately. For instance, a high number of new products with low sales is not necessarily problematic, in the sense that linking old and new GTINs hardly affects a price index in such situations because of their low expenditure shares.

The proposed measure of dynamics is defined as follows at GTIN level:

$$
\frac{\sum_{i \in G_{0, t}} q_{i, t}}{\sum_{i \in G_{t}} q_{i, t}} .
$$

The numerator is equal to the number of items sold in month $t$ that were also sold in the base month, and the denominator is equal to the total number of items sold in month $t$. It is easy to see that this measure is equal to 1 when there are no new items in month $t$, while it decreases when the sales quantities of new items increase. High values of the ratio therefore mean that the existing items prevail in the sales; in other words, the items sold in month $t$ match well with the items sold in the base month. For this reason, Expression (1) will be referred to as the 'degree of product match' in month $t$.

Other choices could be made for different aspects, such as a different base period and to include disappearing products as well. Admittedly, a better choice for the base period would be to take the whole previous year instead of a single month. Products may leave temporarily. A longer period would therefore be recommendable for seasonal products. But for non-seasonal items we do not expect significant differences, as was also noted in Chessa (2018, 23-25). 
In the above definition of product match, adding the quantities sold for disappearing items that were still sold in the base month to the denominator of Expression (1) would not influence the results when comparing different stratification schemes, since the denominator would be the same in every scheme. In other definitions of product match, such as the version based on numbers of products in Chessa (2018), disappearing items had negligible effect on the results.

Examples of product match are shown in Figure 1 for the four types of products mentioned above. The graphs clearly illustrate how much product match can vary across different types of products. Rates of churn are relatively low for milk, cheese and eggs. Most items that were sold in the base month are still sold at the end of a year, as the existing items dominate the sales. The shares of existing items in the sales quantities for hair care drop to about $70 \%$ at the end of each year, so that new items account for about $30 \%$. In this case, we are less confident of choosing GTINs as products. Relaunches are known to occur in this market segment (Chessa 2013).

The other two product types, televisions and infant garments, show extremely low product match values at the end of each year. Item turnover reaches very high levels in the course of a year. Almost entirely new product lines are introduced each year, which practically replace the previous ones. Infant garments are influenced by fashion trends, which may offer an explanation for the high turnover rates and the rapidly decreasing product match.

Traditional bilateral matched model approaches are hard to use at GTIN level under such circumstances, because of the poor continuity of GTINs over time. This is also the case for more sophisticated methods like multilateral methods, since these methods are not able to identify price changes either when relaunches occur. A separate method is developed for handling this problem, which is the purpose of MARS.
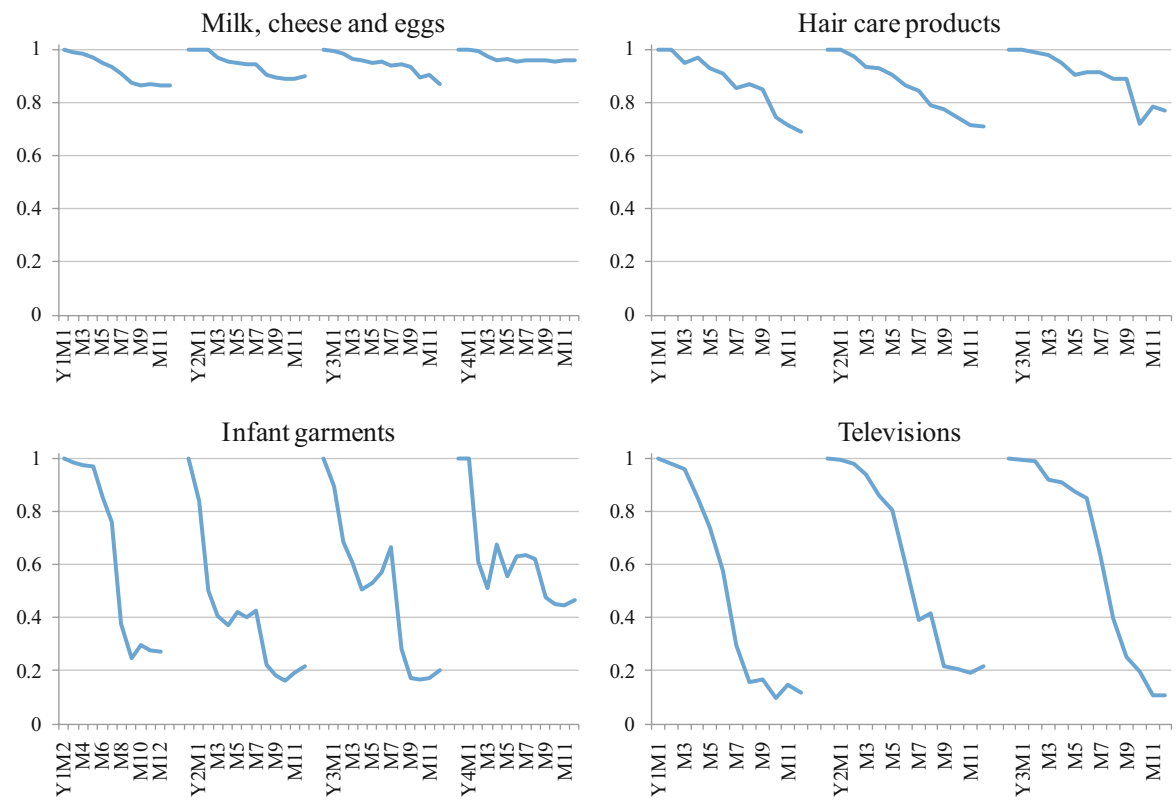

Fig. 1. Degree of product match for four product categories. 
Figure 2 shows price indices when each GTIN is taken as a separate product. These indices are calculated with the 'QU method', a term that is used to denote a family of 'quality adjusted' or 'generalised' unit value methods (Von Auer 2014). The multilateral Geary-Khamis method is an example of the class of QU methods; it is used for processing transaction data in the Dutch CPI since January 2016 and is also the index method used in this article (Chessa 2016). The terms QU and Geary-Khamis are used interchangeably in this article for the same index method.

Making no distinction between GTINs represents the other extreme of the stratification spectrum. In that case, all GTINs would be considered of the same quality. Expenditures and sales quantities are summed over all GTINs within a product category. The ratio yields a weighted average price, known as 'unit value' (ILO et al. 2004). The unit value indices are shown as well in Figure 2.

The graphs show considerable differences between the two indices, especially for televisions. Existing models usually decrease in price after being introduced. New models are often more expensive than their predecessors. The index at GTIN level does not consider any of these higher prices as price changes with respect to older models. This explains why this index decreases. Higher prices of new models are seen as price increases from a unit value perspective, which explains the behaviour of the unit value index. New products may have higher prices because of relaunches, but also because they differ in terms of quality. Shifts in buying behaviour towards more expensive, higher quality products are also considered as price increases by the unit value. Similar explanations for the differences can be given for the other three product groups.

Although the indices represent two extreme cases of product stratification, the differences nevertheless make clear that product definition may have a substantial impact
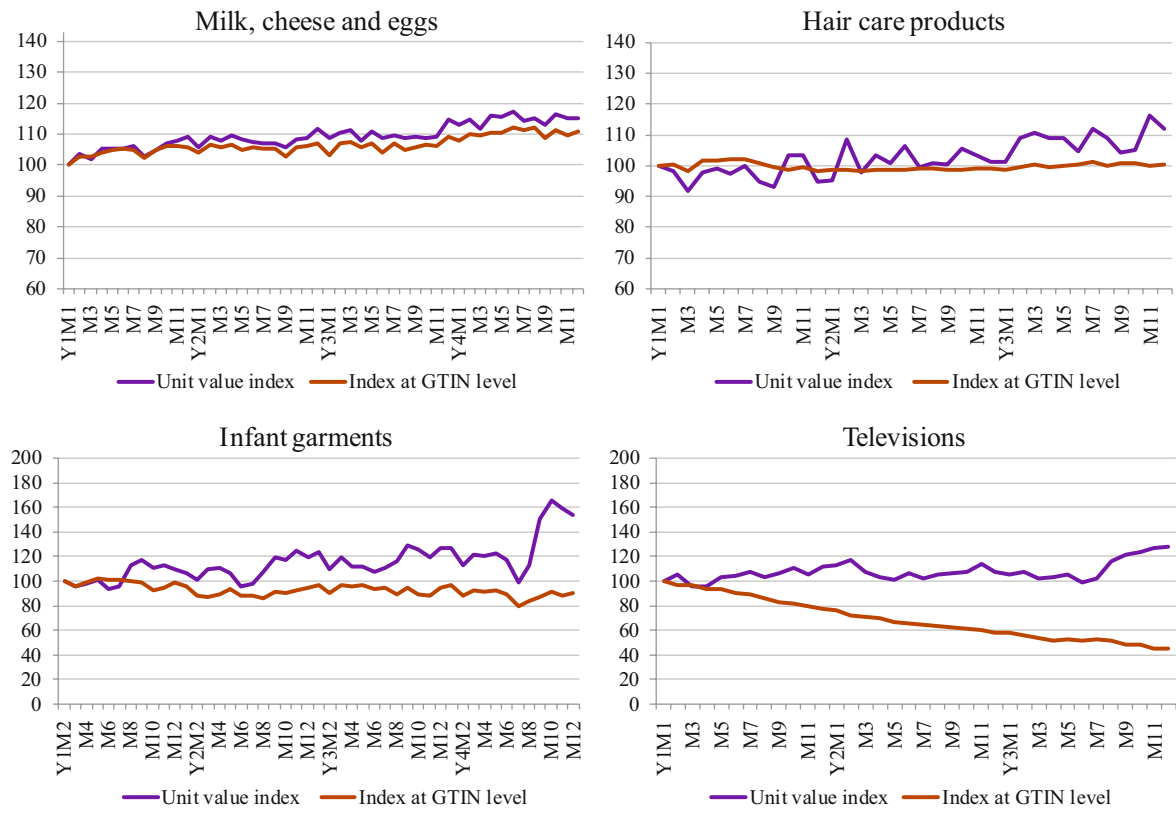

Fig. 2. Price indices at GTIN level and unit value indices for the four product categories (1st month $=100)$. 
on a price index. We are therefore dealing with an influential choice aspect, which requires a balanced and efficient method in order to make careful choices.

\section{Product Definition With MARS}

\subsection{Preliminary Remarks and Terminology}

We start this section by introducing some terminology. The term item was already introduced at the beginning of this article, which is used here interchangeably with GTIN. Different items, that is, with different barcodes but not necessarily in terms of quality, may have to be linked when relaunches occur. The more generic term product is introduced to denote a set of one or more GTINs, which share certain quality characteristics. A product can therefore also be viewed as a combination of characteristics. The latter term is used here as a specific 'value' of the more generic term attribute or variable. For example, 'screen size' is an attribute of televisions, and ' 42 inch' is a specific characteristic.

Items are subdivided into products, and the set of all products forms a partition of GTINs. The term stratification is used in this article as well for partition, where 'partition' is the formal mathematical term. In a partition, each item is assigned to exactly one product, such that products are pairwise disjoint (different products do not have items in common). GTINs may also be chosen as different products, as was illustrated in the previous section, so the set of GTINs is one of the possible partitions. In the examples with unit values there is only one product, which contains all GTINs of a product category.

GTINs should provide a suitable level of stratification for product categories with high degrees of product match. GTINs also represent the most detailed level of product homogeneity in transaction (scanner) data sets. GTINs can therefore be considered as a serious stratification candidate for milk, cheese and eggs. We will return to this in Section 4, when the results of the method MARS are presented. How to select a suitable level of stratification for product categories with low degrees of product match at GTIN level is less obvious. Broader defined products will increase product match, but homogeneity may be adversely affected.

There are different ways of partitioning GTINs into products. Available attributes of GTINs can be used for this purpose. Different selections and combinations of attributes give rise to different partitions. Each product in a partition contains GTINs with the same characteristics. For example, the attributes brand, screen size and screen type yield one partition of the set of televisions. The characteristics 'Samsung', 'between 51 and 59 inch' and 'Ultra HD' define a specific product of this partition. The GTINs within each product are then considered to be of the same or comparable quality. An attribute may be selected or not. This means that a set of GTINs can be partitioned into $2^{n}$ ways for $n$ attributes, to which the partition with GTINs as distinct products can be added (thus yielding $2^{n}+1$ partitions).

\subsection{Formalisation of MARS}

From the introduction to this section it may be clear that numerous ways of partitioning a set of GTINs exist, each of which may have a different impact on product match and homogeneity. The aim is to find a method that balances these two properties in an optimal 
way in some sense. Measures of product match and homogeneity will be set up in order to operationalise this idea. The two measures are eventually combined, which allows evaluation and ranking of GTIN partitions.

First, some notation is introduced in addition to the notation already used in Section 2. We denote a partition by $K$ and use $k$ to indicate an element of a partition, which we have called a 'product'. In theory, different partitions $K_{t}$ can be defined each month $t$, for example by changing the set of attributes. However, such dynamic cases are highly complex and are probably not yet well-understood in terms of price index calculation. This study therefore deals with situations where products, once defined, are kept fixed for some period. A time window of 13 months would be in line with CPI convention, as product definitions are typically reviewed and possibly revised at the end of each year. We can therefore drop the time dimension from the notation for partitions.

We denote the degree of product match for a partition $K$ in month $t$ with respect to the base month by $\mu_{t}^{K}$ and the degree of product homogeneity by $R_{t}^{K}$. It is useful to consider desirable properties for these two measures. Attribute selection and product definition are in fact new subjects in the processing of large electronic data sets for index calculation, so that an initial attempt is made below at defining properties.

- Property 1. For two partitions $K$ and $K^{\prime}$ such that $K^{\prime}$ is a refinement of $K$, so every element of $K^{\prime}$ is a subset of an element of $K$, the degree of product match of the refinement $K^{\prime}$ cannot be larger than the product match of $K$, that is: $\mu_{t}^{K^{\prime}} \leq \mu_{t}^{K}$.

- Property 2. For two partitions $K$ and $K^{\prime}$ such that $K^{\prime}$ is a refinement of $K$, the refinement $K^{\prime}$ is at least as homogeneous as $K$. In formal terms, we have $R_{t}^{K^{\prime}} \geq R_{t}^{K}$ for all $t$.

It is reasonable to expect that broader defined products will increase product match, or at least stay the same, while the opposite is the case for homogeneity. This is in fact what the two properties say. Measures that satisfy both properties are defined below.

\subsubsection{Product Match}

Expression (1) applies to GTINs as separate products, so a generalisation is needed. We introduce $K_{0, t} \subseteq K$ for the set of products that are sold both in base month 0 and a second month $t$, with $t \geq 0$. In practical applications, month 0 will usually be December of the previous year and $t$ a month in a 13-month window that runs until December of the present year. Let $q_{t}^{k}$ denote the number of items sold for product $k$ in month $t$; in formal terms: $q_{t}^{k}=\sum_{i \in G_{t}} q_{i, t} 1_{k}(i)$, where the indicator function $1_{k}(i)$ takes the value 1 if item $i$ belongs to product $k$ and is equal to 0 otherwise. The degree of product match of partition $K$ in month $t$ is defined as follows:

$$
\mu_{t}^{K}=\frac{\sum_{k \in K_{0, t}} q_{t}^{k}}{\sum_{i \in G_{t}} q_{i, t}}
$$

It is easily verified that this measure satisfies Property 1 . Note that $0 \leq \mu_{t}^{K} \leq 1$ for all $K$. 


\subsubsection{Product Homogeneity}

A homogeneous product is a product that consists of items of the same quality. Finding a measure for the homogeneity of a set of different products boils down to finding a method that expresses their quality differences. Hedonics is an approach that comes to mind when reflecting on this complex problem. Although this class of methods has been broadly studied, it is certainly not without limitations (Chessa et al. 2017).

Index methods usually express differences among products in terms of prices. This article also takes item prices to set up a measure of product homogeneity. Alternative choices have not yet been studied; some suggestions for further research are mentioned in Subsection 5.3. We introduce the following notation for prices. Let the price of item $i$ in month $t$ be denoted by $p_{i, t}$ and let $\bar{p}_{t}^{k}$ denote the unit value for product $k$ in month $t$, that is:

$$
\bar{p}_{t}^{k}=\frac{\sum_{i \in G_{t}} p_{i, t} q_{i, t} 1_{k}(i)}{\sum_{i \in G_{t}} q_{i, t} 1_{k}(i)},
$$

where the denominator is equivalent to $q_{t}^{k}$. The unit value over all items in month $t$ is denoted by $\bar{p}_{t}$ :

$$
\bar{p}_{t}=\frac{\sum_{i \in G_{t}} p_{i, t} q_{i, t}}{\sum_{i \in G_{t}} q_{i, t}} .
$$

MARS uses the proportion of explained variance in product prices, relative to the total variance in item prices, as a measure of product homogeneity. The contribution of each product or item is weighted by the quantities sold. This yields the following weighted $\mathrm{R}$ squared measure:

$$
R_{t}^{K}=\frac{\sum_{k \in K} q_{t}^{k}\left(\bar{p}_{t}^{k}-\bar{p}_{t}\right)^{2}}{\sum_{i \in G_{t}} q_{i, t}\left(p_{i, t}-\bar{p}_{t}\right)^{2}}
$$

More precisely, this is in fact a measure of heterogeneity between products. The complementary measure uses the price variance of GTINs within products. We want this measure to be as low as possible and, consequently, the explained variance as high as possible. Higher values of $R_{t}^{K}$ thus denote better homogeneity.

Note that $R_{t}^{K}=0$ when all items are combined into one product and $R_{t}^{K}=1$ when each item is a separate product. Expression (5) satisfies Property 2. Together with the previously mentioned properties, this implies that $0 \leq R_{t}^{K} \leq 1$ for all $K$.

An alternative homogeneity measure could be defined by using coefficients of variation of the products in a partition. These statistics are commonly used in price statistics, not only in the CPI for data analyses, but also in PPPs. However, it can be shown that coefficients of variation do not satisfy Property 2 in general. 


\subsubsection{MARS: Combining Product Match and Homogeneity}

The method MARS aims at evaluating and ranking item partitions. To this end, the measures of homogeneity and product match will be combined. We thus obtain an $\mathrm{R}$ squared measure that is adjusted for the degree of product match, which explains the acronym MARS. Some guidance on suitable functions for the combined measure could be provided by considering properties of rank orderings of partitions.

Transaction data sets, but also other data sources like web scraped data or traditionally collected data, are usually incomplete. For example, the available product variables are a subset of attributes that characterise items, and data sets are usually delivered by retailers in some aggregate form (e.g. aggregated over outlets). The ranking of partitions should not be affected by the degree of detail and aggregation of the data. These considerations motivate the following property.

- Property 3. For any two sets of partitions $\mathcal{K}^{\prime}$ and $\mathcal{K}$ of the same set of items, with $\mathcal{K}^{\prime} \subset \mathcal{K}$, the ordering of partitions on $\mathcal{K}^{\prime}$ should be preserved on larger sets $\mathcal{K}$ that include $\mathcal{K}^{\prime}$.

Property 3 has direct implications for the form of the combined measure of homogeneity and product match. For instance, an arithmetic mean of Expressions (2) and (5) does not necessarily satisfy Property 3. A multiplicative form does satisfy this property, which is the choice made for the method MARS. In relation to this it is useful to note that Expressions (2) and (5) represent measurements on different ratio scales. Item partitions can be compared and ordered in a meaningful way by taking the product of the homogeneity and product match measures. In this article, $\mathrm{R}$ squared and degree of product match are thus combined as follows, for every month $t$ :

$$
M_{t}^{K}=R_{t}^{K} \mu_{t}^{K}
$$

A multiplicative function also has the characteristic that partitions with either low values for $\mathrm{R}$ squared or degree of product match will be ranked as unattractive stratification options.

It will be clear that $0 \leq M_{t}^{K} \leq 1$, since Expressions (2) and (5) also have this property. Expression (6) allows us to evaluate and rank item partitions, such that the partition with the highest value of $M_{t}^{K}$ is preferred. MARS yields values in every month $t$, so, in theory, the ranking of partitions may differ from month to month. The values of MARS in different months have to be combined in some way in order to produce one ranking. Different methods can be envisaged, which will be described and compared in Section 4.

One approach to overcome this is to use a price index to deflate prices and then combine the deflated prices of each product over all months. However, we prefer to stick to the approach proposed in this article, since the addition of a price index would make the method more complex, computationally more intensive and also dependent on index method. The method presented in this article can be combined with any index method, which is a major advantage since different index methods are normally used in the CPI for different forms of price collection.

A separate remark is made for the partition where all items are combined into one product (unit value case). The multiplicative form of measure (6) implies that $M_{t}^{K}=0$ for 
all $t$, which means that the single product partition will always be rejected. If this is found to be a limitation, then a simple remedy could be to increase both the numerator and the denominator of Expression (5) by some constant, say 1. This yields a monotonic transformation of R-squared, which therefore still satisfies Property 2, takes values in $(0$, 1] and preserves the value 1 for the GTIN level. Also Property 3 is still satisfied. However, in practice we do not expect that we have to use modified measures.

\subsubsection{Example}

We illustrate MARS with an example with a small number of items and only one month of data. Consider three GTINs, say A, B and C. The prices and quantities of the GTINs in some month are given in Table 1. The 'status' of each GTIN is also given: GTIN A was sold in the base month and is still sold but is about to leave at a reduced price, GTIN B is new and GTIN C is a regularly sold item. GTIN B could be seen as a relaunch of GTIN A.

We could also specify attributes in order to construct partitions. But, for simplicity, attributes and characteristics are excluded from this example since the number of GTINs is very small. Three GTINs can be partitioned in five ways:

1. A-B-C: each GTIN is a different product,

2. AB-C: GTINs A and B are combined into one product (AB), while $\mathrm{C}$ is a separate product,

3. A-BC: $\mathrm{B}$ and $\mathrm{C}$ are combined into one product, while $\mathrm{A}$ is a separate product,

4. AC-B: $\mathrm{A}$ and $\mathrm{C}$ are combined into one product, while $\mathrm{B}$ is a separate product, and

5. ABC: the three GTINs are combined into one product.

The results are shown in Table 2. A clear preference emerges for partition $A B-C$, in which exiting GTIN A is linked to new GTIN B, while 'persisting' GTIN C is treated as a separate product. This partition maximises the degree of product match, while it makes a minor concession in terms of homogeneity. We can thus say that MARS has picked up the relaunch. Product match is also maximised by linking new GTIN B to C, but this partition (A-BC) greatly affects homogeneity.

Table 1. Prices, quantities and status of the three GTINs.

\begin{tabular}{lrrl}
\hline GTIN & Price & Quantity & Status \\
\hline A & 2.00 & 1 & Exiting \\
B & 4.00 & 20 & New \\
C & 2.00 & 40 & Sold in both months, not exiting \\
\hline
\end{tabular}

Table 2. Results of MARS for the five partitions.

\begin{tabular}{lccc}
\hline Partition & R squared & Product match & Combined \\
\hline A-B-C & 1 & 0.672 & 0.672 \\
AB-C & 0.929 & 1 & 0.929 \\
A-BC & 0.008 & 1 & 0.008 \\
AC-B & 1 & 0.672 & 0.672 \\
ABC & 0 & 1 & 0 \\
\hline
\end{tabular}




\section{Results for Different Product Types}

This section presents the results of MARS for the data of the four product categories that were introduced in Section 2. The method is applied to each of the three or four years of data. The product attributes that are available in the four transaction data sets are used to set up partitions, which are evaluated and ranked with MARS, including the partition in which each GTIN is a separate product.

In this article, products are defined either by combinations of product characteristics or as GTINs. A mixture of these two choices, by using existing GTINs as products when these are not about to disappear and attributes for linking disappearing and new GTINs, is also interesting. This option results in tighter products. As the corresponding price indices hardly differ from those that will follow in this section, this hybrid option is omitted. Details can be found in Chessa (2019, 14-16).

The available product attributes are shown in Table 3. Several remarks can be made about the attributes. GTIN classifiers denote GTIN groups defined by retailers. This information is also contained in the data, which is used by NSIs to facilitate the mapping from GTIN to COICOP in the CPI. The most detailed classifiers could also be used as additional attributes, which is done in this study. For example, the broadest of the two classifiers of milk, cheese and eggs contains seven classes. The second classifier is a further refinement. For example, 'dairy beverages' is subdivided into milk, buttermilk and several other groups. The classifiers for hair care distinguish between conditioners and shampoo, and the most detailed classifier mainly by hair type (e.g. normal, dry).

Apart from package volume, the other attributes are categorical variables. This is also the case for screen size (televisions), which is expressed as a range (e.g. from 28 to 32 inch). Screen type in fact means display technology (e.g. OLED, Ultra HD). Because of the level of detail used by the retailer to specify colours for clothing, we decided to compress colours into three classes (white, black and coloured). The other attributes were used as specified by the retailers. The example with colour shows that different choices can also be made in terms of how the specified characteristics (i.e. the different colours) are used. Using them as specified or further compressing the range of colours increases the number of partitions. This illustrates again the complexity of the problem of product definition from a combinatorial perspective.

Partitions have been set up by using the product attributes in Table 3. These partitions, together with the partition in which every GTIN represents a separate product, are evaluated and ranked with MARS. The method MARS yields a score in each month for every partition. The monthly scores are combined into a single score, which is eventually used to rank the partitions.

Table 3. Product attributes in the four transaction data sets.

\begin{tabular}{ll}
\hline Product category & Variables/attributes \\
\hline $\begin{array}{l}\text { Milk, cheese, eggs } \\
\text { Infant garments }\end{array}$ & $\begin{array}{c}\text { Brand, package volume, 2 GTIN classifiers } \\
\text { Type of garment, volume (\#items), fabric, } \\
\text { sleeve length, colour, fit, size }\end{array}$ \\
$\begin{array}{l}\text { Hair care } \\
\text { Televisions }\end{array}$ & Brand, package volume, 2 GTIN classifiers \\
\hline
\end{tabular}


In this section, the MARS scores of the last three months are taken to compute an average score for each partition. The idea behind this choice is that the effects of churn become more apparent towards the end of a year, since the products that are sold in the base month will dominate sales in the first months of a year. The results are shown in Table 4. An obvious question is whether the rankings of the partitions will change when the three-month period is extended. We will come back to this later in this section.

These results invite us to make a number of remarks:

- The partition 'GTINs as products' is only chosen for milk, cheese and eggs, and emerges as the best partition in each year,

- The results tell us that products for clothing, hair care and televisions should be defined by sets of attributes. The three product categories are characterised by moderately to rapidly decreasing degrees of product match at GTIN level (high rates of churn), and

- For milk, cheese and eggs, hair care and televisions, the selection of attributes is quite stable over the years. The results for infant garments show more variability.

These findings probably summarise what results could be expected beforehand in a broad sense. GTINs are an appropriate choice for milk, cheese and eggs, and also for other COICOP 01 items (not shown here) but not for the other product categories because of the higher rates of churn.

GTINs are not selected as products for hair care, infant garments and televisions. The degrees of product match at GTIN level are very low for infant garments and televisions in the second half of each year (Figure 1). New GTINs may thus have to be linked to GTINs that leave the stores. The results show that in most cases a small set of attributes is selected and used for linking. The number of attributes selected in the third and fourth year for infant garments is clearly larger than in the first two years. Apparently, the degree of product match increases for tighter defined products in the third and fourth year.

The variability in the sets of selected attributes raises an important question on how MARS could be used in CPI production. In practice, decisions about product definition are made for the next year. The item partitions in this section are obtained by using the data of the corresponding year. It is therefore of tremendous practical interest to know the extent to which the price indices presented in this section change when the best partition of the current year is used to compute an index with the data of the next year. We will return to this question in Subsection 5.2.

Figure 3 shows the monthly MARS scores for various GTIN partitions. Several remarks can be made based on these graphs. First, the GTIN scheme has the highest MARS scores

Table 4. Partitions selected by MARS for the four product categories in each year.

\begin{tabular}{|c|c|c|c|c|}
\hline Product category & Year 1 & Year 2 & Year 3 & Year 4 \\
\hline Milk, cheese, eggs & GTINs & GTINs & GTINs & GTINs \\
\hline Infant garments & $\begin{array}{l}\text { Type, volume, } \\
\text { sleeve length }\end{array}$ & $\begin{array}{l}\text { Type, volume, } \\
\text { colour, fit }\end{array}$ & All attributes & $\begin{array}{l}\text { All attributes, } \\
\text { except colour }\end{array}$ \\
\hline Hair care & All attributes & Brand, volume & Brand, volume & \\
\hline Televisions & $\begin{array}{l}\text { Screen size, } \\
\text { screen type }\end{array}$ & $\begin{array}{l}\text { Screen size, screen } \\
\text { type, brand }\end{array}$ & $\begin{array}{l}\text { Screen size, screen } \\
\text { type, brand }\end{array}$ & \\
\hline
\end{tabular}



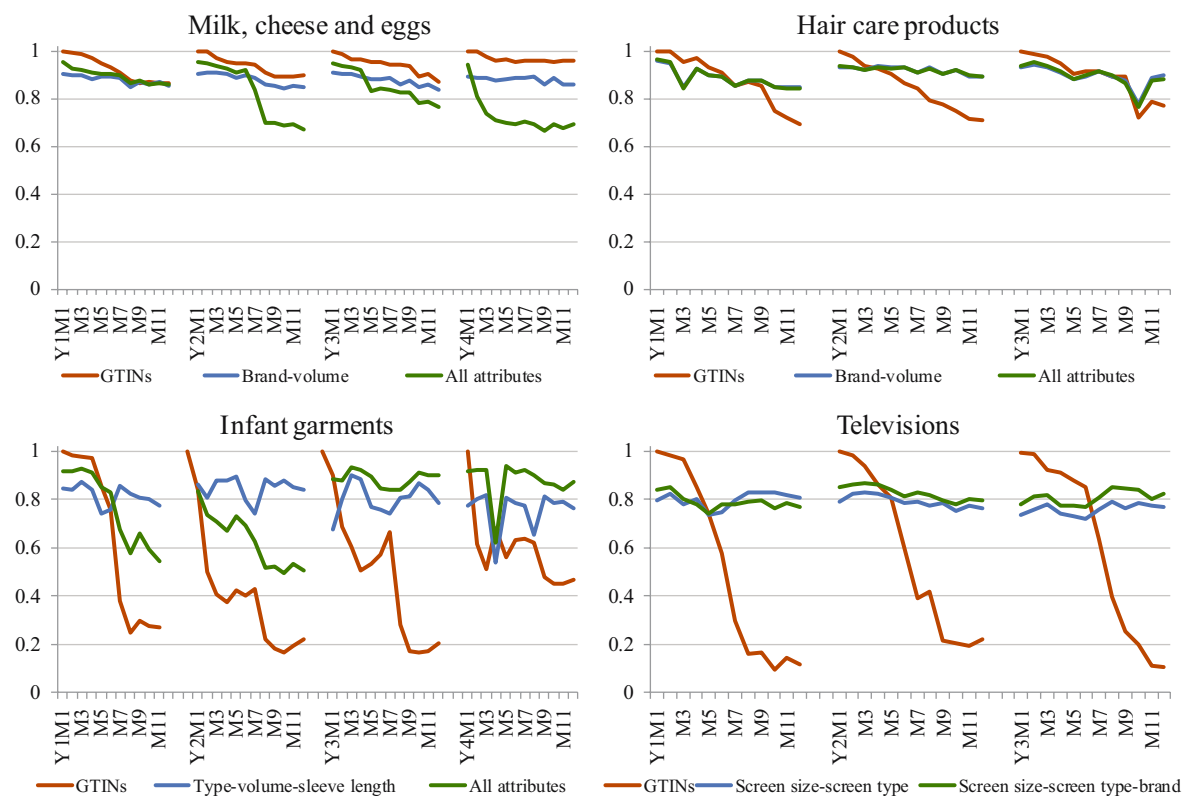

Fig. 3. MARS scores for different partitions of the four product categories.

in the first months of each year, which confirms the idea expressed above to shift the focus towards the second half of a year in terms of evaluating and ranking partitions. As was noted previously, this is most apparent for hair care, infant garments and televisions because of the high churn rates.

Second, the MARS scores in the second half of each year lead to the same ordering of the partitions in almost all months in the graphs of Figure 3. This means that extending the three-month period up to six months in order to calculate an average MARS score for every partition would hardly change the results for the data in this article. The same conclusion was obtained in a previous study (Chessa 2018).

Third, the partition that results from selecting all attributes for milk, cheese and eggs shows a particular behaviour in the second and fourth year. It reaches much lower MARS scores than the other two partitions in the second half of these two years. Product match turns out to be much lower than at GTIN level. This should not happen, since the GTIN level is a refinement of any partition based on attributes. This means that Property 1 in Subsection 3.2 is violated. The rapid decreases in product match for the 'all attributes' partition are caused by changes in the names of some GTIN classifiers. Products with changed names are considered as new products, which in reality are existing products with new classifier descriptions.

This example shows the practical usefulness of Property 1 and that MARS can also be used as a data monitoring tool. More generally, this also shows that stratifying items by attributes requires high quality metadata, which have to be checked each month prior to compiling the CPI. For example, retailers may decide to abbreviate names of GTIN classifiers (e.g. "bt. milk" instead of "buttermilk"). Such changes in names have to be identified in a timely manner also in order to assign GTINs to their correct COICOP. 
The price indices that correspond with the highest ranked partitions in each year (Table 4) are compared with the index for GTINs as products and the unit value index. The first two indices are calculated with the previously mentioned QU method (end of Section 2). This is done on 13-month time windows. Longer-term index series are obtained by linking the 13-month indices to the indices in the base month (December of the previous year).

The three indices are shown in Figure 4. The index for milk, cheese and eggs at GTIN level is the same as the index for the best partition. The indices for the best partitions for the other product categories show large differences with the indices at GTIN level and the unit value index. The partitions are based on attributes, which enable picking up price differences between new and exiting GTINs with the same characteristics. This results in higher indices compared with the indices at GTIN level for hair care, infant garments and televisions.

The indices for the best partitions for infant garments and televisions also differ substantially from the unit value indices. The differences between these two indices for hair care are smaller. Although products are defined as combinations of characteristics, this does not mean that we should expect the corresponding indices to behave like a unit value index. Products may be tightly defined in such partitions, as is indicated by the figures in Table 5. In relation to this, note that MARS allows new and disappearing products to occur also at broader product levels than GTIN.

In some cases, the average number of GTINs per product is quite large. This may be caused by the relatively small number of attributes in the transaction data sets. On the other hand, it cannot be excluded that broader groups are sufficiently homogeneous. The
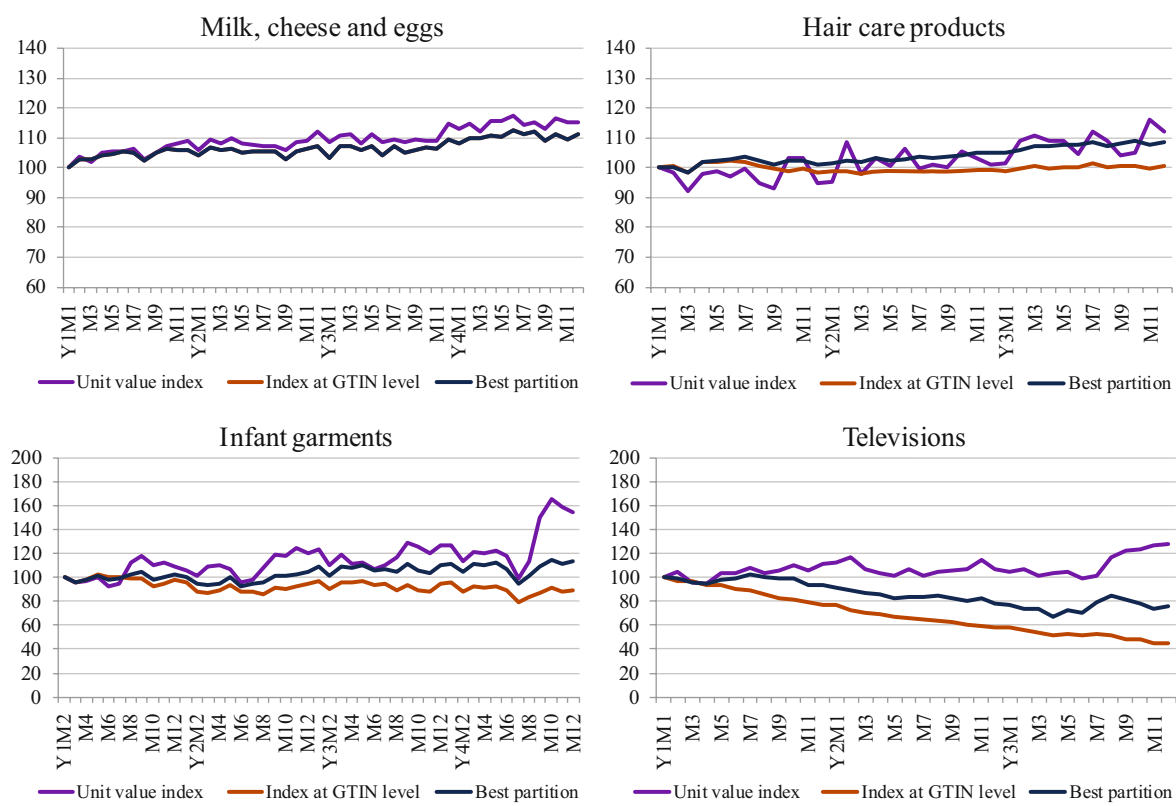

Fig. 4. Price indices for the yearly best partitions, compared with the indices at GTIN level and the unit value indices. 
Table 5. Average number of GTINs per product.

\begin{tabular}{lcccc}
\hline Product category & Year 1 & Year 2 & Year 3 & Year 4 \\
\hline Milk, cheese, eggs & \multicolumn{1}{c}{1} & 1 & 1 & 1 \\
Infant garments & 20.3 & 24.6 & 4.2 & 5.8 \\
Hair care & 3.0 & 5.4 & 6.0 & \\
Televisions & 21.7 & 6.6 & 7.1 & \\
\hline
\end{tabular}

data for infant garments contain seven attributes. The partition with four attributes reaches high MARS scores and clearly dominates the partition based on all available attributes in the first two years (Figure 3). Intuitively, this could be interpreted as a sign that the clothing data contain enough attributes. This topic will be discussed further in Subsection 5.1.

Apart from the question of whether the available product attributes are sufficient, it is also interesting to ask whether the price indices for the best partitions are stable. Sensitivity analyses were carried out by varying the weights for the two measures of MARS. Homogeneity and product match are in fact assigned the same weight in Expression (6). Tighter products are favoured by increasing the weight of homogeneity.

An increase of this weight from 0.5 to 0.75 , that is, making homogeneity three times more important than product match, hardly changes the results. Brand would be selected for televisions also in the first year and all attributes would be selected for hair care in the second year. No changes were found for the other two product categories. The price indices are not shown since these hardly changed. A previous study on consumer electronics showed stable results for a similar sensitivity analysis (Chessa 2018).

\section{Topics of Further Research}

\subsection{Information on Product Attributes}

This section discusses methodological questions related to the availability of product attributes in transaction data sets. Roughly, two situations may occur: data sets containing a limited number of attributes (the situation in this article) and data sets that contain a large number of attributes. Although transaction data sets typically contain up to four or five attributes, there are NSIs, like Statistics Netherlands, that recently received data sets with tens of product attributes. The request and acquisition of more detailed data is driven by a growing experience with the use of transaction data in the CPI and retailers' growing familiarity with data acquisition and usage by NSIs. Maintaining relationships between NSIs and retailers is an important part of this process.

The two situations concerning data availability lead to the following questions:

(1) In cases with small numbers of product attributes, is the number of attributes sufficient for defining products? Are there ways to establish whether more attributes are needed?

(2) If the number of attributes is large, say in the order of tens of attributes, the number of stratification schemes quickly becomes too large to enumerate and evaluate. The question is how large solution spaces could be handled efficiently. 


\subsubsection{Small Numbers of Attributes}

First, note that the question about sufficiency of product attributes becomes more relevant when the rate of churn increases (i.e. when product match decreases). GTINs are suited as products for stable assortments, in which case the question is not relevant. The COICOP milk, cheese and eggs is an example of this (Figure 3). MARS results in a selection of attributes for the other three product categories. Not all attributes are needed in most years, which could be interpreted as an indication that the available attributes are sufficient and also that products can be defined with a limited number of attributes. However, Chessa $(2019,17)$ showed that adding attributes from web scraped data for televisions leads to a different set of attributes and a significantly different price index.

This example shows that the question on data sufficiency is a difficult one. Suggestions to deal with this problem are given below:

- Adding attributes from a second source, such as web scraping. Building and maintaining web scrapers and analysing data requires time. If the main purpose is to use product characteristics, then it should be sufficient to scrape such information once a month. If information about a lot of attributes is available on websites, then the question below has to be addressed, on how variables can be selected from a large set of product attributes,

- Alternative approaches could be considered if web scraping is not feasible in the short term. A practical approach could be to ask CPI analysts to inspect whether products with the highest expenditure shares should be stratified further. More refined stratifications could then be evaluated with MARS,

- The second suggestion may be time-consuming. Another approach could be to generate product refinements in an automatic way by introducing a dummy attribute with a number of 'values' (dummy characteristics). The idea then is to draw a value for every GTIN at random, which leads to a further product refinement. This process can be repeated a number of times and the resulting stratification schemes can be evaluated with MARS. Improved MARS scores indicate a need for collecting information about additional attributes. A brief illustration of this idea can be found in Chessa $(2018,32-33)$.

\subsubsection{Large Numbers of Attributes}

NSIs have various possibilities to extend sets of product attributes in transaction data. This can be done by web scraping, but also by requesting additional information from retailers. Data sets containing tens of attributes are already being received by NSIs, which raises the question as to how such numbers can be handled for product definition. Stratification schemes based on, say up to about 10 attributes, can all be evaluated separately, but this quickly becomes inefficient for larger numbers of attributes.

The problem addressed in this article is a combinatorial optimisation problem, in which Expression (6) is the objective function to be maximised over a set of item partitions. A number of optimisation methods are worth investigating:

- The best-first search method selects the variable that gives the largest improvement in the objective function at each iteration step. The procedure is repeated with the 
remaining variables until the objective function does not improve anymore (Russell and Norvig 2003). This method does not guarantee a global optimum, but is attractive for its simplicity and speed, as $n$ variables require at most $n(n+1) / 2$ evaluations,

- Branch and bound searches for an optimal stratification scheme by setting up a rooted tree, where a branch specifies whether a variable is either selected or not. Each node, or subtree, represents a subset of stratification schemes, with the full set contained in the root. Upper bounds of the objective function to be maximised are calculated at each node, which are compared with the best solution found so far. If an upper bound is smaller than the current value of the objective function then the corresponding subtree can be excluded from further exploration. For details, see Land and Doig (1960) and Little et al. (1963), and

- Another method that may be worth considering is simulated annealing (Kirkpatrick et al. 1983; Granville et al. 1994). This is an iterative method that decides whether to stay in the current 'state' (i.e. stratification scheme) or move to a new state with a certain probability. The transition probabilities are specified such that a move to a scheme with a lower MARS score than the current one has positive probability, in order to avoid being trapped in local optima. The probability of accepting a scheme with a lower MARS score is gradually decreased as the number of iteration steps increases.

To get a first impression of the performance of best-first search (BFS), the method was applied to the four data sets used in this article, although cases with more attributes are obviously more interesting. The method trivially gives the same results for milk, cheese and eggs, as the GTIN level gives the highest MARS scores. However, BFS gives the same results also for the other three data sets in each year. The data on infant garments contain seven attributes. The selection of attributes changes from year to year (Table 4), but BFS yields the same results.

BFS can be applied in a simple and efficient way, and the excellent results obtained for the data sets in this article make it a very appealing and promising method. As it may get stuck in a local maximum, it is worth combining BFS with the other two methods mentioned above. BFS could be applied at each node of the tree in a branch and bound method. BFS can also be used to generate an initial state in a simulated annealing algorithm and could also be used in subsequent stages. These combined strategies are worth investigating in future research.

\subsection{Practical Issues}

An important question is how MARS could be applied in a balanced way in production. Different factors should be considered, such as:

- The 'retail dimension'. Should MARS be applied to each retail chain separately or is it possible to combine the data of different retail chains for the same product category?,

- The 'product dimension'. Also in this case the question is which level of aggregation would be feasible under resources and time constraints, and

- The 'time dimension'. This refers to the frequency of maintenance of product definitions during a year. 
Applying MARS to each retail chain for the same aggregate is probably very time consuming from a production perspective. People working in a production environment have a limited amount of time that they can spend on analysing the results of product stratification. To give an idea, there are more than 50 COICOPs at the most detailed publication level (five-digit COICOP) for COICOP 01 (food and non-alcoholic beverages). The number of applications of MARS would thus become quite large only for supermarkets. Applying MARS to the pooled data of all retail chains for the same product category seems a better idea. Retail chains should be distinguished when applying MARS, which can be done by treating retail chain as a distinct attribute.

A similar problem arises in the product dimension. Applying MARS below COICOP-5 level would result in a large number of applications. The proposal of combining data of retail chains and applying MARS at COICOP-5 level therefore seems a balanced proposal.

Another important question is how often product definitions should be checked and possibly revised during a year. Will once a year, at the end of a year, be sufficient? Or should more checks be carried out? Once a year is most compatible with current CPI routine and also saves time. A higher frequency has the advantage of timely signalling sudden changes in product assortments, like changes in product churn.

The results in Section 4 showed that product definitions may change over time. Those for infant garments even change each year. Product definitions were established by making use of the data of the same year. In practice, decisions have to be taken for the next year. An interesting question therefore is to what extent the indices will change if these are based on the product definitions established in the preceding year. Figure 5 shows that the resulting price indices are accurate. The results also suggest that it may be sufficient to check product definitions at the end of a year. Some monitoring, say after half a year, is nevertheless useful.
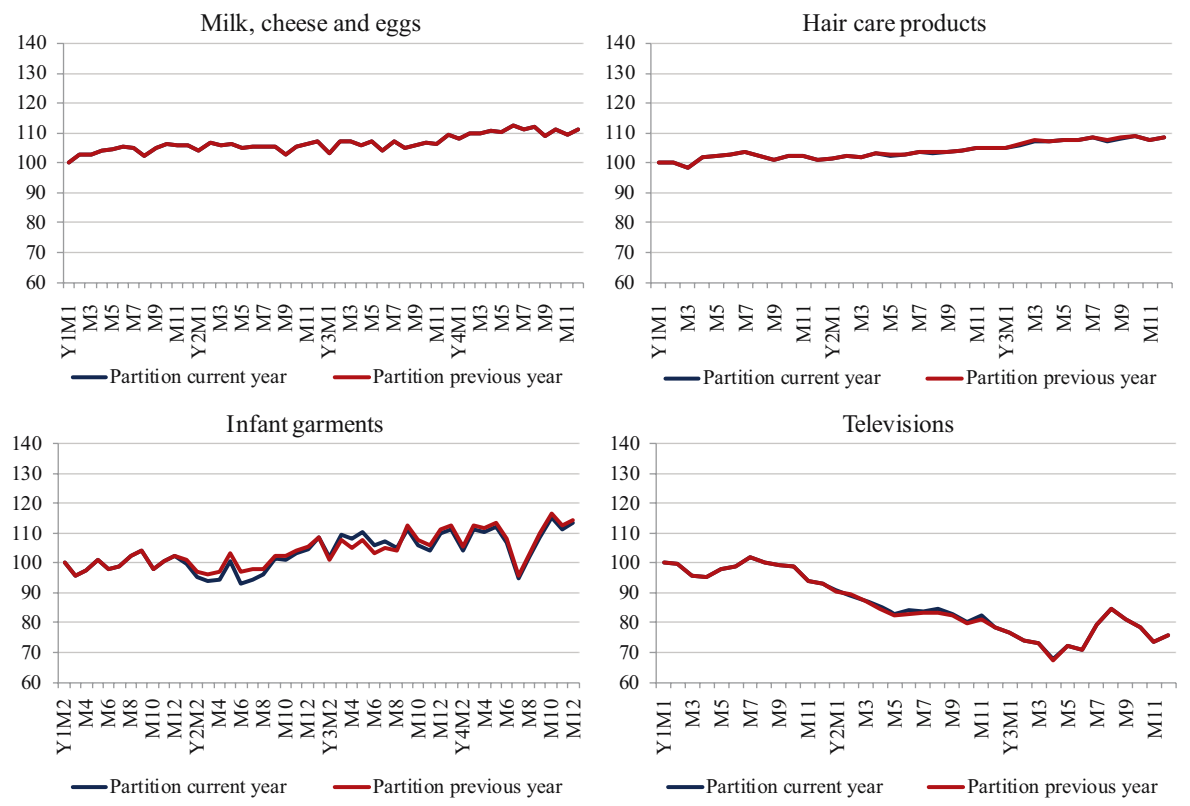

Fig. 5. Price indices when using the product definitions of the preceding year. 


\subsection{Possible Alternatives for MARS}

Taken from a more general perspective, the idea behind MARS is to find similarity measures for items within strata (homogeneity) and over time (product match). A number of specific choices lead to the method MARS:

- MARS does not make direct use of attribute values in the homogeneity measure, but rather uses a mapping of the vector of characteristics of each item onto a onedimensional variable, which is the item's price in this study,

- The (squared) Euclidean distance of an item to the average price of its corresponding stratum is taken here as an item's similarity measure, which are combined for different items into an overall homogeneity measure, and

- The measure of product match makes use of the numbers of items sold.

The reasons for choosing item prices and numbers of items sold were given in Section 2 and Subsection 3.2. However, these choices may also have shortcomings:

- Prices and numbers sold may vary substantially from month to month, for example during sales periods. Such variations may affect the ranking of item partitions, and

- The choice of item prices has the advantage that distance measures between items can be easily defined and calculated, but on the other hand results in indirect comparisons of the characteristics of different items.

The results in Section 4 showed that the ranking of partitions remains stable within a year for each product type, based on the MARS scores in the last six months of each year. The variability in the rankings increases over subsequent years. The set of selected attributes may change from year to year, but this could be a sign of new developments in a market segment (electronics is a typical example). Such developments should be detected and accounted for in both product definition and index calculation.

Nevertheless, it is worth studying alternatives for the choices made in MARS. Inspiration may be gained from the vast literature on cluster analysis and similarity measurement. The following suggestions may serve as a starting point:

- One direction could be to study more robust choices in the current method. For example, the number of items sold could be replaced by the number of items within products. This choice may be less sensitive to relaunches, but has the advantage of being more robust in sales periods. An additional advantage is that the number of items within item strata can also be applied to other data sources, like web scraped data,

- Alternative distance measures could be considered, such as the maximum norm instead of the Euclidean norm, and

- Alternatives to item prices in the homogeneity measure could be considered, by directly comparing the vectors of characteristics of different items. This increases the complexity of the problem, since product attributes may reflect different types of measurement (e.g. numerical, categorical or binary). Transaction data contain different types of attributes, which is also the case for the data used in this study (Table 3, Section 4). The literature on similarity measurement contains studies that focus on a specific type of variable, like numerical variables (Mafteiu-Scai 2013) and 
categorical variables (Boriah et al. 2008), and also studies that combine similarity measures for different types of variables (Hoffmann et al. 2015).

\section{Final Remarks}

Price collection is traditionally carried out by following prices of representative items that satisfy product definitions. This approach is feasible for relatively small product samples. So, historically, the problem of product definition is not new. But it needs a new, more efficient treatment when NSIs consider switching to big electronic data sets and aim at processing a significant part of such data sets or even to their full extent.

The method MARS facilitates such a transition, as this method can be used to automate the problem of product definition to a high degree. The need for a higher degree of automation and data processing was not the only motivation behind the development of MARS. The method also contributes to formalising the notion of "product", which has been an unfilled gap in price statistics so far.

MARS yields tightly defined products for stable assortments, while products are defined as broader strata, based on common characteristics, as churn rates increase. The latter result was also found in a previous study on consumer electronics (Chessa 2018). MARS is able to identify relaunches, so that price changes associated with this phenomenon will be picked up. The results in this article and in Chessa (2018) also show that the price indices for the stratification scheme with the highest MARS scores are quite stable. The sensitivity analyses that have been carried out hardly affect the results of MARS and the corresponding indices.

MARS has been extensively applied to transaction data. Web scraping is a rapidly growing field, which motivates applying MARS also to web scraped data. This requires finding proxies for quantities sold (e.g. number of scraped product prices per month, see Chessa and Griffioen 2019). The suggestions mentioned in Subsection 5.1 for dealing with small and large numbers of product attributes and the suggestions in Subsection 5.3 for alternative versions of MARS are also worth investigating in future research.

\section{References}

ABS. 2017. "Making Greater Use of Transactions Data to Compile the Consumer Price Index." 15th Meeting of the Ottawa Group on Price Indices, 10-12 May 2017, Eltville am Rhein, Germany. Available at: https://www.bundesbank.de/en/homepage/makinggreater-use-of-transactions-data-to-compile-the-consumer-price-index-australia635722 (accessed November 2019).

Bilius, Å., O. Ståhl, and C. Tongur. 2018. "Coverage Bias and the Effect of Re-launches in Scanner Data: A Coffee Index." Meeting of the Group of Experts on Consumer Price Indices, 7-9 May 2018, Geneva, Switzerland. Available at: https://www.unece.org/fileadmin/DAM/stats/documents/ece/ces/ge.22/2018/Sweden_poster_2_ppt.pdf (accessed November 2019).

Boriah, S., V. Chandola, and V. Kumar. 2008. "Similarity Measures for Categorical Data: A Comparative Evaluation." In Proceedings of the 2008 SIAM International Conference on Data Mining, 24-26 April 2008, Atlanta, Georgia, United States: 243-254. DOI: https://doi.org/10.1137/1.9781611972788.22. 
Chessa, A.G. 2013. "Comparing Scanner Data and Survey Data for Measuring Price Change of Drugstore Articles." Workshop on Scanner Data for HICP, 26-27 September 2013, Lisbon, Portugal. Available at: https://www.ine.pt/xportal/xmain?xpi $\mathrm{d}=$ INE\&xpgid $=$ ine_sem_lista\&tipo $=$ r\&detalhe $=165101941$ (accessed November 2019).

Chessa, A.G. 2016. "A New Methodology for Processing Scanner Data in the Dutch CPI." Eurostat Review on National Accounts and Macroeconomic Indicators 2016(1): 49-69. Available at: https://ec.europa.eu/eurostat/cros/content/new-methodology-processingscanner-data-dutch-cpi-antonio-g-chessa_en (accessed November 2019).

Chessa, A.G. 2018. Product Definition and Index Calculation with MARS-QU: Applications to Consumer Electronics. The Hague: Statistics Netherlands. Available at: https://circabc.europa.eu/sd/a/16b279bd-04d1-44bd-8972-a537f09f7c59/Report\%20 Grant\%202017-18\%20Objective\%201C.PDF.pdf (accessed May 2021).

Chessa, A.G. 2019. "MARS: A Method for Defining Products and Linking Barcodes of Item Relaunches." 16th Meeting of the Ottawa Group on Price Indices, 8-10 May 2019, Rio de Janeiro, Brazil. Available at: https://eventos.fgv.br/sites/eventos.fgv.br/files/arquivos/u161/product_definition_with_mars_chessa_og19.pdf (accessed November 2019).

Chessa, A.G., and R. Griffioen. 2019. "Comparing Scanner Data and Web Scraped Data for Consumer Price Indices.” Economie et Statistique/Economics and Statistics 509: 49-68. DOI: https://doi.org/10.24187/ecostat.2019.509.1984.

Chessa, A.G., J. Verburg, and L. Willenborg. 2017. "A Comparison of Price Index Methods for Scanner Data." 15th Meeting of the Ottawa Group on Price Indices, 10-12 May 2017, Eltville am Rhein, Germany. Available at: http://www.ottawagroup.org/ Ottawa/ottawagroup.nsf/4a256353001af3ed4b2562bb00121564/1ab31c25da944ff5ca2 $5822 \mathrm{c} 00757 \mathrm{f} 87 /$ FILE/A\%20comparison\%20of\%20price\%20index \%20methods $\% 20 \mathrm{f}$ or\%20scanner\%20data\%20-Antonio\%20Chessa, \%20Johan\%20Verburg, \%20Leon \% 20Willenborg\%20-Paper.pdf (accessed November 2019).

De Haan, J., and H.A. van der Grient. 2011. "Eliminating Chain Drift in Price Indices Based on Scanner Data.” Journal of Econometrics 161: 36-46. DOI: https://doi.org/10. 1016/j.jeconom.2010.09.004.

Diewert, W.E., and K.J. Fox. 2017. Substitution Bias in Multilateral Methods for CPI Construction Using Scanner Data. Vancouver: The University of British Columbia. Discussion paper 17-02. Available at: https://www.ottawagroup.org/Ottawa/ottawagroup.nsf/4a256353001af3ed4b2562bb00121564/1ab31c25da944ff5ca25822c00757f87/ \$FILE/Substitution\%20bias\%20in\%20multilateral\%20methods\%20for\%20CPI\%20construction\%20using\%20scanner\%20data\%20-Erwin\%20Diewert, \%20Kevin\%20Fox\% 20-Paper.pdf (accessed May 2021).

Granville, V., M. Krivanek, and J.-P. Rasson. 1994. "Simulated Annealing: A Proof of Convergence." IEEE Transactions on Pattern Analysis and Machine Intelligence 16: 652-656. DOI: https://doi.org/10.1109/34.295910.

Hoffmann, U., A. da Silva, and M. Carvalho. 2015. "Finding Similar Products in Ecommerce Sites Based on Attributes." In Proceedings of the 9th Alberto Mendelzon International Workshop on Foundations of Data Management, 6-8 May 2015, Lima, Peru. Available at: http://ceur-ws.org/Vol-1378/ (accessed November 2019). 
Hov, K., and R. Johannessen. 2018. "Using Scanner Data for Sports Equipment.” Meeting of the Group of Experts on Consumer Price Indices, 7-9 May 2018, Geneva, Switzerland. Available at: https://www.unece.org/fileadmin/DAM/stats/documents/ece/ces/ge.22/2018/Norway_-_session_1.pdf (accessed November 2019).

ILO, IMF, OECD, UNECE, Eurostat, and The World Bank. 2004. Consumer Price Index Manual: Theory and Practice. Geneva: ILO Publications. DOI: https://doi.org/10.5089/ 9787509510148.069.

Ivancic, L., W.E. Diewert, and K.J. Fox. 2011. "Scanner Data, Time Aggregation and the Construction of Price Indexes.” Journal of Econometrics 161: 24-35. DOI: https://doi. org/10.1016/j.jeconom.2010.09.003.

Keating, J., and M. Murtagh. 2018. "Quality Adjustment in the Irish CPI." Meeting of the Group of Experts on Consumer Price Indices, 7-9 May 2018, Geneva, Switzerland. Available at: https://www.unece.org/fileadmin/DAM/stats/documents/ece/ces/ge.22/2018/Ireland.pdf (accessed November 2019).

Kirkpatrick, S., C.D. Gelatt Jr, and M.P. Vecchi. 1983. "Optimization by Simulated Annealing.” Science 220 (4598): 671-680. DOI: https://doi.org/10.1126/science.220. 4598.671.

Krsinich, F. 2014. "The FEWS Index: Fixed Effects with a Window Splice - NonRevisable Quality-Adjusted Price Indices with No Characteristic Information.” Meeting of the Group of Experts on Consumer Price Indices, 26-28 May 2014, Geneva, Switzerland. Available at: https://www.unece.org/fileadmin/DAM/stats/documents/ece/ces/ge.22/2014/New_Zealand_-_FEWS.pdf (accessed November 2019).

Land, A.H., and A.G. Doig. 1960. "An Automatic Method of Solving Discrete Programming Problems." Econometrica 28: 497-520. DOI: https://doi.org/10.2307/ 1910129.

Little, J.D.C., K.G. Murty, D.W. Sweeney, and C. Karel. 1963. "An Algorithm for the Traveling Salesman Problem.” Operations Research 11: 972-989. DOI: https://doi.org/ $10.2307 / 1910129$.

Mafteiu-Scai, L.O. 2013. "A New Dissimilarity Measure between Feature-Vectors." International Journal of Computer Applications 64: 39-44. DOI: https://doi.org/10. 5120/10730-5734.

Russell, S.J., and P. Norvig. 2003. Artificial Intelligence: A Modern Approach (2nd ed.). Upper Saddle River, New Jersey: Prentice Hall. DOI: https://doi.org/10.1017/s0269888 900007724.

Van Loon, K., and D. Roels. 2018. “Integrating Big Data in the Belgian CPI.” Meeting of the Group of Experts on Consumer Price Indices, 7-9 May 2018, Geneva, Switzerland. Available at: https:/www.unece.org/fileadmin/DAM/stats/documents/ece/ces/ge.22/2018/Belgium.pdf (accessed November 2019).

Von Auer, L. 2014. "The Generalized Unit Value Index Family." Review of Income and Wealth 60: 843-861. DOI: https://doi.org/10.1111/roiw.12042.

Received June 2019

Revised December 2019

Accepted April 2020 